\title{
ON SYMMETRIC DETERMINANTS
}

\author{
BY W. V. PARKER
}

In a former paper* the writer proved the following theorem:

THEOREM A. If $D=\left|a_{i j}\right|$ is a symmetric determinant of order $n>4$ with $a_{i j}$ real and $a_{i i}=0,(i=1,2, \cdots, n)$, and if all fourthorder principal minors of $D$ are zero, then $D$ vanishes.

The purpose of this note is to give some results which are obtained immediately from this theorem and which are in one sense a generalization of this theorem.

Suppose $D$ is a symmetric determinant of order $n>4$, with real elements, in which all principal minors of order $n-1$ and also all principal minors of order $n-4$ are zero. If $D^{\prime}=\left|A_{i j}\right|$ is the adjoint of $D$, then $A_{i i}=0,(i=1,2, \cdots, n)$. Each fourthorder principal minor of $D^{\prime}$ is equal to the product of $D^{3}$ by a principal minor of $D$ of order $n-4$. $\uparrow$ Therefore $D^{\prime}$ satisfies the conditions of Theorem $\mathrm{A}$ and hence is zero. But $D^{\prime}=D^{n-1}$ and hence $D$ is also zero and we have the following theorem:

THEOREM 1. If $D$ is a symmetric determinant of order $n>4$, with real elements, in which all principal minors of order $n-1$ and also all principal minors of order $n-4$ are zero, then $D$ vanishes.

Suppose $D$ is a symmetric determinant of order $n>4$, with real elements, in which all principal minors of some order $k>3$ and also all principal minors of order $k-3$ are zero. Let $M$ be any $(k+1)$-rowed principal minor of $D,(M=D$ if $n=5)$, then $M$ is a determinant satisfying the conditions of Theorem 1 and hence $M$ is zero. Therefore, in $D$, all principal minors of order $k$ and also all principal minors of order $k+1$ are zero, hence $D$ is of rank $k-1$ or less. $\ddagger$ We have thus proved the following theorem:

* On real symmetric determinants whose principal diagonal elements are zero, this Bulletin, vol. 38 (1932), pp. 259-262. See also, On symmetric determinants, American Mathematical Monthly, vol. 41 (1934), pp. 174-178.

$\dagger$ Bôcher, Introduction to Higher Algebra, p. 31.

$\ddagger$ Bôcher, loc. cit., page 57, Theorem 2. 
THEOREM 2. If $D$ is a symmetric determinant of order $n>4$, with real elements, in which all principal minors of some order $k>3$ and also all principal minors of order $k-3$ are zero, then $D$ is of rank $k-1$ or less.

If $n>5$, and $k<n-1$ the minors of Theorem 2 may be divided into two complementary sets such that if all minors of either set are zero the determinant vanishes. This division into sets may be done in $n$ different ways.

Suppose $D$ is a symmetric determinant of order $n>5$, with real elements, and $M$ is a principal minor of $D$ of order $n-1$. If all principal minors of $M$ of some order $k>3$ and also all principal minors of $M$ of order $k-3$ are zero, then $M$ is of rank $k-1$ or less by Theorem 2. Let us suppose now that $M$ is in the upper left hand corner of $D$ and expand $D$ according to the products of the elements of the last row and the last column. We get

$$
D=a_{n n} M-\sum_{i, j=1}^{n-1} a_{n i} a_{j n} \alpha_{i j},
$$

where $\alpha_{i j}$ is the cofactor of $a_{i j}$ in $M$. If now we make the further restriction that $k$ be less than $n-1$, then, since the rank of $M$ is $k-1$ or less, each $\alpha_{i j}=0$ and consequently $D=0$. We have, therefore, the following result:

TheOREM 3. If $D$ is a symmetric determinant of order $n>5$, with real elements, and $M$ is a principal minor of $D$ of order $n-1$, and if all principal minors of $M$ of some order $k, 3<k<n-1$, and also all principal minors of $M$ of order $k-3$ are zero, then $D$ vanishes.

Suppose $D$ is a symmetric determinant of order $n>5$, with real elements, and that $M$ is a principal minor of $D$ of order $n-1$. Suppose also that all principal minors of $D$ of some order $n-t$ and also all principal minors of $D$ of order $n-t+3,(t>3)$, which are not minors of $M$, are zero. We may assume further, without loss of generality, that $M$ is in the upper left hand corner of $D$. Let $D^{\prime}$ be the adjoint of $D$ and $M^{\prime}$ be the minor of $D^{\prime}$ corresponding to $M$ in $D$. Any principal minor of $M^{\prime}$ of order $t$ (of order $t-3$ ) is equal to the product of $D^{t-1}\left(D^{t-4}\right)$ by the complement in $D$ of the corresponding minor in $M$. This com- 
plementary minor is a minor of $D$ of order $n-t(n-t+3)$ and is not a minor of $M$ and hence is zero by hypothesis. Therefore $M^{\prime}$ is a symmetric determinant of order $n-1>4$, with real elements, in which all principal minors of some order $t>3$ and also all principal minors of order $t-3$ are zero, and hence $M^{\prime}$ is of rank $t-1$ or less by Theorem 2 . If we make the further restric tion that $t$ be less than $n-1$ we find, by expanding $D^{\prime}$ according to the products of the elements of the last row and the last column, that $D^{\prime}$ is zero. But $D^{\prime}=D^{n-1}$ and hence $D$ is zero also.

If we write $n-t+3=k$, since $3<t<n-1$, we have $4<k<n$ and hence the truth of the following theorem is apparent:

THEOREM 4. If $D$ is a symmetric determinant of order $n>5$, with real elements, and $M$ is any principal minor of $D$ of order $n-1$, and if all principal minors of $D$ of some order $k>4$ and also all principal minors of $D$ of order $k-3$, which are not minors of $M$, are zero, then $D$ vanishes.

In a second paper the writer* proved a theorem stated as follows:

TheOREM B. If $D=\left|a_{i j}\right|$ is a symmetric determinant of order $n>5$, in which $a_{i i}=0,(i=1,2, \cdots, n)$, and $M$ is any principal minor of $D$ of order $n-1$, then if all fourth order principal minors of $D$ which are not minors of $M$ are zero, $D$ vanishes.

From this theorem we see that the restriction that the elements of $D$ be real is not necessary in Theorem A when $n$ is greater than five. Consequently the theorems of this paper may be extended to include determinants with complex elements. Theorem 1 is true for complex elements if $n>5$. Theorem 2 is true for complex elements if $n>5$ and $k>4$. Theorems 3 and 4 are true for complex elements if $n>6$ and $4<k<n-1$.

\section{Louisiana State University}

*A theorem on symmetric determinants, this Bulletin, vol. 38 (1932), pp. 545-550. 